\title{
Effect of Maternal Smoking on Fetal Catecholamine Concentrations at Birth
}

\author{
CHERYL A. ONCKEN, KERRIE M. HENRY, WINSTON A. CAMPBELL, CYNTHIA M. KUHN, \\ THEODORE A. SLOTKIN, AND HENRY R. KRANZLER
}

\begin{abstract}
Department of Medicine [C.A.O.], Department of Obstetrics and Gynecology [C.A.O., W.A.C.], Department of Psychiatry [H.R.K.], University of Connecticut Health Center, Farmington, Connecticut, U.S.A.; Department of Obstetrics and Gynecology, Hartford Hospital, Hartford, Connecticut, U.S.A. [K.M.H.]; Department of Pharmacology and Cancer Biology, Duke University Medical Center, Durham,
\end{abstract} North Carolina, U.S.A. [C.M.K., T.A.S.]

\begin{abstract}
The aim of this study was to compare catecholamine concentrations in the fetal umbilical artery cord blood from the offspring of smokers versus the offspring of nonsmokers. Pregnant women who were self-identified as smokers $(\geq 10$ cigarettes per day throughout pregnancy) or nonsmokers were recruited for study participation. Maternal blood was collected for cotinine concentrations. Umbilical artery cord blood was collected at delivery for arterial $\mathrm{pH}$ and catecholamine concentrations. Cord blood was obtained from 51 subjects, including 21 smokers and 30 nonsmokers. Median epinephrine concentrations $[304 \mathrm{pg} / \mathrm{mL}$ versus $597 \mathrm{pg} / \mathrm{mL}$ (Mann-Whitney $U=170 ; p=0.006$ )] and median norepinephrine concentrations $[3148 \mathrm{pg} / \mathrm{mL}$ versus $6558 \mathrm{pg} / \mathrm{mL}$ (Mann-Whitney $U=191 ; p=0.006$ )] were significantly lower in smokers compared with nonsmokers, respectively. After controlling for gestational age, route of delivery, and arterial $\mathrm{pH}$,
\end{abstract}

\section{ABSTRACT}

log-transformed epinephrine concentrations between smokers and nonsmokers were statistically significant $(p=0.03)$, with a similar trend for log-transformed norepinephrine concentrations $(p=0.07)$. Analyses of the data using cotinine $<20 \mathrm{ng} / \mathrm{mL}$ to classify nonsmokers also showed differences in epinephrine concentrations between groups $(p=0.02)$. These results are consistent with results from animal studies showing that catecholamine concentrations may be affected by prenatal nicotine exposure. Further studies are needed to validate these findings and to examine the specific mechanism by which these differences may arise. (Pediatr Res 53: 119-124, 2003)

\section{Abbreviation \\ SIDS, sudden infant death syndrome}

Maternal smoking is one of the most important causes of poor pregnancy outcomes in developed countries. Maternal smoking increases the risk of spontaneous abortion, low birth weight, premature delivery, and infant death from perinatal disorders and from SIDS (1-3). One of the most dramatic effects of maternal smoking is a tripling of the risk of SIDS, a tragic syndrome that claims thousands of infant lives each year (1). Although the epidemiologic association between smoking and these disorders is well established, the mechanism by which smoking contributes to these health risks is not well understood.

SIDS may result from cardiorespiratory failure of the infant during a hypoxic episode, as in sleep apnea (4). If this is the

Received February 27, 2002; accepted July 23, 2002.

Correspondence: Cheryl Oncken, M.D., M.P.H., Department of Medicine, MC 3940, University of Connecticut School of Medicine, 263 Farmington Ave., Farmington, CT 06030-3940, U.S.A.; e-mail oncken@nso2.uchc.edu

Supported, in part, by National Institutes of Health grants AA03510, DA15167, AA00239 (H.R.K.), RR06192 (General Clinical Research Center Grant of the University of Connecticut Health Center), DA13334, CA89053, and DA14247.

DOI: 10.1203/01.PDR.0000042443.65546.E1 case, animal research suggests that smoking could contribute to the increased risk of SIDS through changes in cell differentiation within the adrenal medulla. The ability to survive hypoxic challenge requires responses mediated by circulating catecholamines, which the fetal or neonatal adrenal medulla does via an autonomous mechanism that is independent of CNS innervation (5). This mechanism disappears with further cell differentiation, triggered by the development of innervation, and the consequent stimulation of the nicotinic cholinergic receptors on the chromaffin cells by the neurotransmitter, acetylcholine (6). Nicotine mimics acetylcholine, leading to premature loss of the fetal/neonatal response. Consistent with this hypothesis, the offspring of pregnant rats administered nicotine throughout gestation were more likely to die during a postnatal hypoxic challenge than were controls (7). The cause of death in the nicotine-treated group in this study was an inability of the offspring to mount an adequate adrenal response.

To examine the relevance of this model to SIDS in humans, we conducted a pilot study to determine whether maternal 
smoking affects fetal adrenal catecholamine release in response to the hypoxia associated with delivery. Parturition was chosen as the time to assess fetal catecholamine release because, during the birthing process, the fetus is subjected to a relative hypoxemia. Specifically, during labor, the arterial blood $\mathrm{Po}_{2}$ decreases from $35 \mathrm{~mm} \mathrm{Hg}$ to $23 \mathrm{~mm} \mathrm{Hg}$ (8). If there is intrapartum asphyxia, the decrease in $\mathrm{Po}_{2}$ is even greater. We hypothesized that, compared with the offspring of nonsmokers, infants born to women who smoke during pregnancy will show a blunted adrenal catecholamine response to normal labor hypoxia or to labor associated with fetal distress (i.e. severe hypoxia with acidosis).

\section{MATERIALS AND METHODS}

Overview. The study protocol was approved by the Institutional Review Board at the University of Connecticut Health Center and Hartford Hospital. We obtained written informed consent from all subjects at the time of hospital admission to labor and delivery. Women were identified through self-report as either smokers or nonsmokers based on the nursing intake interview and an initial research interview.

Subjects. We recruited women who were $\geq 18$ y of age and who either smoked $\geq 10$ cigarettes per day throughout pregnancy (smokers) or who did not smoke during pregnancy (nonsmokers). Women were asked if they had smoked any cigarettes at all during their pregnancy. Those who reported not smoking at all (i.e. not one single puff) were recruited for the nonsmoking group. We did not routinely inquire whether the subject was exposed to second-hand smoke either at home or in the workplace. We excluded women who reported alcohol use during pregnancy in excess of one drink per month, who reported using illicit or nonprescribed drugs during pregnancy, who used tobacco products other than cigarettes or nicotine replacement during pregnancy, or who had an uncontrolled endocrine disorder.

Procedures. Basic demographic and clinical data were obtained through a research interview or from review of the clinical chart. The following information was obtained: age, race, education, number of cigarettes smoked per day, gestational age at delivery, pregnancy history, length of labor, anesthetics received during labor (local, epidural, spinal, or general anesthesia), Apgar scores at 1 and $5 \mathrm{~min}$, type of delivery [normal spontaneous vaginal delivery, operative vaginal delivery, elective cesarean delivery, or indicated cesarean delivery (failed labor progression or fetal distress)], and maternal medical conditions and medications. Although we sought to match smokers and nonsmokers on gestational age (within $1 \mathrm{wk}$ ), route of delivery, and any maternal medical conditions, it was not always possible to find a match. Samples were obtained from 21 smokers and 30 nonsmokers, and potential confounding factors were controlled statistically.

Maternal blood $(6 \mathrm{~mL})$ was drawn to measure cotinine [the major metabolite of nicotine and a representative measure of overall nicotine intake (9)]. Umbilical artery cord blood was obtained at the time of delivery. The umbilical artery carries blood from the fetal circulation back to the placenta and is a better measure of fetal hypoxia, acidosis, and catecholamine secretion than blood from the fetal vein (10). Thus, samples for the main analyses were taken from this artery, although, in some instances, a separate sample was also obtained from the fetal vein. The umbilical cord was double clamped approximately 2 inches from the umbilicus, using a plastic cord clamp proximal to the infant, and a Kelly clamp approximately 1 inch distal to the cord clamp. The cord was cut between these two clamps and the infant handed off to nursing personnel for care. Approximately 6 inches distal to the Kelly clamp, two more clamps were placed 1 inch apart. The cord was cut between these two distal clamps to obtain a segment of cord for umbilical cord blood analyses $\left(\mathrm{pH}, \mathrm{PCO}_{2}, \mathrm{Po}_{2}\right)$. Cord blood samples for catecholamine assay were drawn from the remaining segment of cord that was still attached to the undelivered placenta. The blood was drawn into an EDTA tube and kept on ice. Cord blood samples were spun in a refrigerated centrifuge and plasma was removed, transferred to a tube containing glutathione $\left(60 \mathrm{mg} / \mathrm{mL}\right.$ solution) and stored at $-70^{\circ} \mathrm{C}$ until analysis.

Assays. Catecholamines (epinephrine and norepinephrine) were analyzed by HPLC with electrochemical detection at Duke University (11). Cotinine was measured by gas chromatography using a nitrogen phosphorous detector at Hennepin County Medical Center, Minneapolis, MN, U.S.A. (12).

Sample size estimation. Because no data exist to estimate the effect of smoking on fetal catecholamine concentrations at delivery, we used data on catecholamine concentrations among preterm and full-term infants to estimate the sample size required to provide adequate statistical power (13). This calculation is justifiable, because preterm delivery and low birth weight are also risk factors for SIDS (14). Because some infants are born of uneventful deliveries, whereas others may be more stressed, we used data from infants born under both conditions to provide a more conservative estimate of effect size. The mean arterial catecholamine concentration for fullterm infants was $62.1 \mathrm{nmol} / \mathrm{L}(\mathrm{SD}=34.9 \mathrm{nmol} / \mathrm{L})$ and for preterm infants was $37.8 \mathrm{~nm} / \mathrm{L}(\mathrm{SD}=4.1 \mathrm{~nm} / \mathrm{L})$. This yields an effect $(d)=0.88$, which is in the range of large effect sizes (15). In infants where there was evidence of asphyxia at the time of delivery, full-term infants had a mean arterial catecholamine concentration of $218 \mathrm{~nm} / \mathrm{L}(\mathrm{SD}=229.3 \mathrm{~nm} / \mathrm{L})$, whereas preterm infants had a mean catecholamine concentration of $115.2 \mathrm{nmol} / \mathrm{L}(\mathrm{SD}=56.62 \mathrm{nmol} / \mathrm{L})(13)$. These data yield an effect $(\mathrm{d})=0.57$, which in the range of medium effect sizes (15). Therefore, assuming a medium-to-large effect size (d) of approximately 0.72 , a total sample of 43 subjects is required to provide $80 \%$ power, using stepwise linear regression analysis and $\alpha=0.05$.

Data analysis. Data were analyzed using SPSS statistical software (SPSS Inc., Chicago, IL, U.S.A.). ANOVA and $\chi^{2}$ analysis were used to compare smokers and nonsmokers on demographic variables. Medians were used to describe variables that were not normally distributed, and the MannWhitney $U$ test was used to compare median values. Because the main outcome measures (i.e. epinephrine and norepinephrine concentrations) were not normally distributed, they were log-transformed for the main analyses. A correlational analysis was conducted to determine the associations between demo- 
graphic and clinical variables and epinephrine and norepinephrine concentrations. We used hierarchical multiple regression analysis to compare log epinephrine and log norepinephrine in smokers and nonsmokers after controlling for a number of potential confounders: study site, maternal age, education, race, gravida, maternal medical conditions, breech delivery, narcotics, and birth weight and route of delivery. Route of delivery was categorized as normal spontaneous vaginal delivery versus other because most of the deliveries in each group were of this type, and because this category had the lowest mean catecholamine concentrations compared with the other routes of delivery. The aforementioned variables were all entered into the model, however, a variable was later removed if it was not significant and there was no a priori hypothesis that this variable could affect catecholamines. This process was done to recapture the degree of freedom. Analyses were conducted with and without outliers, and because the results were similar, all subjects are included in the final results.

\section{RESULTS}

Cord blood samples and arterial blood gas samples were obtained on 52 subjects; 21 smokers and 31 nonsmokers. One subject with an unusually low concentration of norepinephrine (i.e. $417 \mathrm{pg} / \mathrm{mL}$ ) was excluded from the analyses when review of her chart revealed that she was given terbutaline (a catecholamine agonist) and magnesium sulfate for $3 \mathrm{~d}$ before delivery, and that she received a dose of terbutaline $10 \mathrm{~h}$ before delivery. Unusually low concentrations of norepinephrine in this individual were thought to be the result of suppression of endogenous catecholamine production from an exogenous catecholamine agonist. Although five other subjects had received terbutaline during pregnancy, none had received it within the $3 \mathrm{~d}$ before delivery.

Demographic and clinical features by group. As shown in Table 1, self-reported smokers were younger, less educated, and more likely to be Caucasian than nonsmokers. Smokers also had more prior pregnancies, smaller infants, and infants

Table 1. Demographic characteristics of smokers and nonsmokers

\begin{tabular}{lccl}
\hline & $\begin{array}{c}\text { Smokers } \\
(n=21)\end{array}$ & $\begin{array}{c}\text { Nonsmokers } \\
(n=30)\end{array}$ & $p$-value \\
\hline Site (\% from UCHC) & $12 / 21(57 \%)$ & $12 / 30(40 \%)$ & 0.18 \\
Age (years) & $27 \pm 5$ & $31 \pm 6$ & 0.02 \\
Education (years) & $12 \pm 2$ & $14 \pm 3$ & 0.01 \\
Race (\% Caucasian) & $16 / 21(76 \%)$ & $13 / 30(43 \%)$ & 0.01 \\
Number of cigarettes/day & $17 \pm 8$ & $0 \pm 0$ & 0.0001 \\
Plasma cotinine (ng/mL) & $115 \pm 118$ & $32 \pm 36$ & 0.001 \\
Gravida & $4 \pm 2$ & $3 \pm 2$ & 0.002 \\
Gestational age (wk) & $37 \pm 3$ & $38 \pm 2$ & 0.07 \\
\% NSVD & $13 / 21(62 \%)$ & $12 / 30(40 \%)$ & 0.16 \\
\% Narcotics given during & $8 / 21(38 \%)$ & $11 / 30(37 \%)$ & 0.52 \\
$\quad$ & & & \\
delivery & $1 / 21(5 \%)$ & $3 / 30(10 \%)$ & 0.45 \\
Length of Labor (min) & $442 \pm 402$ & $466 \pm 413$ & 0.84 \\
Birth Weight (grams) & $2675 \pm 645$ & $3396 \pm 652$ & 0.0001 \\
Apgar Score at 1 min & $7.5 \pm 2$ & $8.2 \pm 1$ & 0.12 \\
Apgar Score 5 min & $8.8 \pm 6$ & $8.8 \pm .8$ & 0.85 \\
\hline
\end{tabular}

All values are reported as mean \pm SD. NSVD $=$ Normal spontaneous vaginal delivery. Statistical comparisons based on analysis of variance for continuous measures and $\chi^{2}$ analysis for categorical variables. that were born earlier than those of nonsmokers. Although, as anticipated, smokers had a higher plasma cotinine concentration than nonsmokers, the cotinine concentration in nonsmokers was higher than those usually seen in nonsmokers (i.e. 20 $\mathrm{ng} / \mathrm{mL}$ or less) (16). In both groups, the most common route of delivery was a normal spontaneous vaginal delivery. Among smokers, 13 of $21(62 \%)$ were by normal spontaneous vaginal delivery, 2 of $21(9 \%)$ had elective cesarean delivery, and 6 of $21(29 \%)$ were cesarean delivery for failed labor or fetal distress. Among nonsmokers, 12 of $30(40 \%)$ were by normal spontaneous vaginal delivery, 7 of $30(23 \%)$ had operative vaginal deliveries, 6 of $30(20 \%)$ had elective cesarean delivery, and 5 of $30(17 \%)$ were by cesarean delivery indicated for fetal distress or failed labor progression.

There were no group differences in the percentage of subjects recruited from the two hospitals, the percentage of infants born breech, the percentage of women who received narcotic medication during labor, or Apgar scores at 1 and $5 \mathrm{~min}$. The types of anesthesia were also similar between the two groups. Among smokers, 1 of $21(5 \%)$ had no anesthesia, 15 of 21 (71\%) had epidural anesthesia, 4 of $21(19 \%)$ had spinal anesthesia, and 1 of $21(5 \%)$ had general anesthesia. Among the nonsmokers, 4 of $30(13 \%)$ had no anesthesia, 21 of 30 $(70 \%)$ had epidural anesthesia, 4 of $30(13 \%)$ had spinal anesthesia, and 1 of $30(3 \%)$ had general anesthesia. Nine smokers had a medical condition [asthma, gestational diabetes (each with $n=2$ ); depression, migraine, mild hypertension, HIV infection, Tourette syndrome (each with $n=1$ )], compared with seven nonsmokers with a medical condition [HIV infection $(n=2)$; depression, gestational diabetes, migraine, mild hypertension, $\beta$ thalassemia trait (each with $n=1$ )].

Arterial blood gas and catecholamine concentrations by group. Umbilical cord blood arterial blood gas (ABG) and catecholamine (epinephrine and norepinephrine) concentrations for smokers and nonsmokers are shown in Table 2. Because the distribution of catecholamine concentrations was not normal, both mean and median values are reported. Mean values for $\mathrm{ABG}$ concentrations $\left(\mathrm{pH}, \mathrm{Po}_{2}, \mathrm{P}_{2}\right)$ did not differ significantly by group. As shown in Figure 1, the median concentrations of both epinephrine (Mann-Whitney $U=170$;

Table 2. Cord blood measures in smokers and nonsmokers

\begin{tabular}{|c|c|c|c|}
\hline & $\begin{array}{c}\text { Smokers } \\
(n=21)\end{array}$ & $\begin{array}{c}\text { Nonsmokers } \\
\quad(n=30)\end{array}$ & $p$-value \\
\hline \multicolumn{4}{|l|}{ Arterial $\mathrm{pH}$} \\
\hline Mean \pm SD & $7.27 \pm 0.08$ & $7.27 \pm 0.07$ & 0.77 \\
\hline \multicolumn{4}{|c|}{ Arterial $\mathrm{PO}_{2}(\mathrm{mmHg})$} \\
\hline Mean \pm SD & $22 \pm 15$ & $18 \pm 8$ & 0.25 \\
\hline \multicolumn{4}{|c|}{ Arterial $\mathrm{PCO}_{2}(\mathrm{mmHg})$} \\
\hline Mean \pm SD & $51 \pm 10.5$ & $54 \pm 11$ & 0.39 \\
\hline \multicolumn{4}{|c|}{ Epinephrine $(\mathrm{pg} / \mathrm{mL})$} \\
\hline Median & 304 & 597 & 0.006 \\
\hline Mean \pm SD & $398 \pm 433$ & $1186 \pm 1792$ & \\
\hline \multicolumn{4}{|c|}{ Norepinephrine $(\mathrm{pg} / \mathrm{mL})$} \\
\hline Median & 3148 & 6558 & 0.018 \\
\hline Mean $\pm \mathrm{SD}$ & $6815 \pm 8950$ & $13679 \pm 16759$ & \\
\hline
\end{tabular}

Values are reported as Mean $\pm \mathrm{SD}$. Medians are also reported for nonnormally distributed measures. Statistical comparisons based on analysis of variance for arterial $\mathrm{pH}, \mathrm{P}_{\mathrm{o} 2}$ and $\mathrm{PC}_{\mathrm{o} 2}$, and the Mann-Whitney $\mathrm{U}$ Test for epinephrine and norepinephrine. 


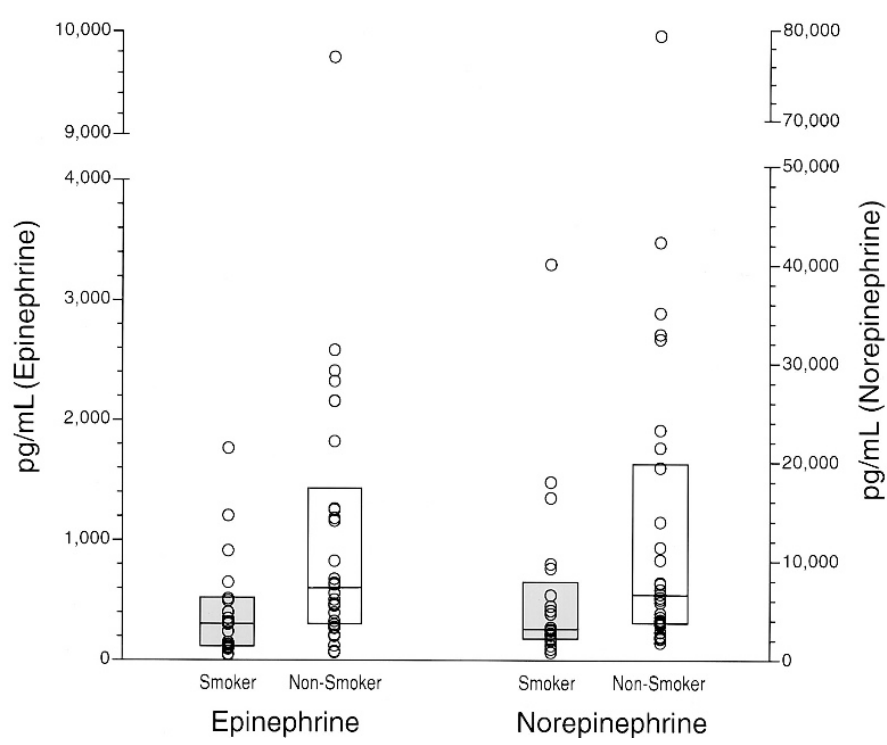

Figure 1. Distribution of catecholamine (epinephrine and norepinephrine) concentrations in smokers and nonsmokers. Box plots represent median values and the 25 th and 75 th percentiles.

$p=0.006$ ) and norepinephrine (Mann-Whitney $U=191 ; p=$ $0.006)$ were significantly lower in smokers versus nonsmokers.

The mean log epinephrine values were $5.4(\mathrm{SD}=1.0)$ and $6.4(\mathrm{SD}=1.1)(p=0.003)$ and the mean log norepinephrine values were $8.3(\mathrm{SD}=1.0)$ and $9.0(\mathrm{SD}=1.0)(p=0.02)$ for smokers and nonsmokers, respectively. A comparison of smokers $(n=21)$ with nonsmokers who had a cotinine concentration of $<20 \mathrm{ng} / \mathrm{mL}$ (i.e. biologically confirmed nonsmokers; $n$ $=13$ ) showed that the mean log epinephrine was $5.5(\mathrm{SD}=$ $1.0)$ and $6.7(\mathrm{SD}=1.1)(p=0.003)$ and $\log$ norepinephrine was $8.3(\mathrm{SD}=1.0)$ and $8.7(\mathrm{SD}=0.7)(p=0.19)$ for smokers and nonsmokers, respectively.

The mean log epinephrine for normal spontaneous vaginal delivery was $5.7(\mathrm{SD}=1.1)$, for elective cesarean delivery was $5.8(\mathrm{SD}=1.3)$, for emergent cesarean delivery was $6.1(\mathrm{SD}=$ 0.7 ), and for operative vaginal delivery was 7.6 ( $\mathrm{SD}=0.8$ ). ANOVA comparing the mean log epinephrine across groups was statistically significant $(p=0.001)$. Posthoc analysis showed that operative vaginal delivery had higher epinephrine concentrations than the other three route of delivery groups $(p$ $<0.05)$. The mean log norepinephrine concentration was 8.3 $(\mathrm{SD}=0.9)$ for normal spontaneous vaginal delivery, 8.6 (SD $=1.1)$ for elective cesarean delivery, $9.1(\mathrm{SD}=0.9)$ for emergent cesarean delivery, and $9.7(\mathrm{SD}=0.8)$ for operative vaginal delivery. ANOVA comparing the mean log norepinephrine concentration across groups was statistically signifi- cant $(p=0.003)$. Posthoc analysis showed that operative vaginal delivery had higher norepinephrine concentrations than normal spontaneous vaginal delivery $(p<0.05)$. There was also a difference between delivery groups in arterial $\mathrm{pH}$. The mean $\mathrm{pH}$ for normal spontaneous vaginal delivery was 7.31 $(\mathrm{SD}=0.01)$, for elective cesarean section it was $7.28(\mathrm{SD}=$ $0.06)$, for emergent cesarean section it was $7.26(\mathrm{SD}=0.05)$, and for operative vaginal delivery it was $7.22(\mathrm{SD}=0.05)$. ANOVA comparing the mean $\mathrm{pH}$ across groups was statistically significant $(p=0.01)$, with posthoc analysis showing that the $\mathrm{pH}$ for normal spontaneous vaginal delivery was higher than that for operative vaginal delivery and elective cesarean section $(p<0.05)$.

Univariate associations between smoking status and other features. There were significant bivariate associations between log epinephrine and log norepinephrine and smoking status $(r$ $=-0.408, p=0.003 ; r=-0.325, p=0.02$, respectively), gestational age $(r=0.455, p=0.001 ; r=0.310, p=0.027$, respectively), and birth weight ( $r=0.426, p=0.002 ; r=$ $0.334, p=0.017$, respectively). Route of delivery was negatively correlated with $\log$ epinephrine $(r=-0.291, p=0.038)$ and norepinephrine $(r=-0.386, p=0.005)$ indicating that normal spontaneous vaginal delivery had lower catecholamine concentrations than other routes of delivery. Log norepinephrine was also negatively correlated with arterial $\mathrm{pH}(r=$ $-0.323, p=0.021)$. Among variables in the analytic model, cotinine was correlated with smoking status $(r=0.466, p=$ $0.001)$, and birth weight was highly correlated with gestational age $(r=0.769, p=0.001)$.

Hierarchical multiple regression. Variables that were retained in the final model included gestational age and route of delivery (Table 3). Arterial $\mathrm{pH}$ was also retained in the equation because of its known effect on catecholamine concentrations. Birth weight, which was highly correlated with gestational age, was not retained in the model. After controlling for gestational age, the addition of birth weight to the model did not change the overall significance of the smoking status variable for either the full sample or the cotinine-validated sample. As shown in Table 3, after controlling for gestational age, route of delivery, and arterial $\mathrm{pH}$, there was a significant difference in epinephrine concentrations between smokers and nonsmokers, irrespective of whether smoking status was based on self-report or cotinine concentration. There was a nonsignificant trend ( $p=0.07$ ) for norepinephrine to vary by selfreport smoking status. However, when smokers were compared with cotinine-validated nonsmokers, there was no effect of smoking status on norepinephrine concentration.

Table 3. Predictors of umbilical artery catecholamine concentrations in smokers vs. nonsmokers: $\beta$ coefficients and significance levels

\begin{tabular}{|c|c|c|c|c|}
\hline Variable & Gestational age & ROD & Arterial $\mathrm{pH}$ & Smoking \\
\hline Log epinephrine (full sample) & $0.46 ; p=0.001$ & $-0.205 ; p=0.12$ & $0.074 ; p=0.64$ & $-0.290 ; p=0.03$ \\
\hline Log epinephrine (cotinine-validated nonsmokers) & $0.44 ; p=0.01$ & $-0.302 ; p=0.06$ & $0.113 ; p=0.55$ & $-0.338 ; p=0.02$ \\
\hline Log norepinephrine (full sample) & $0.31 ; p=0.03$ & $-0.336 ; p=0.02$ & $-0.095 ; p=0.55$ & $-0.253 ; p=0.07$ \\
\hline
\end{tabular}

$p$-Value for the regression models in which study group was a significant predictor. Model compares differences in smokers versus nonsmokers controlling for gestational age (weeks), route of delivery (normal spontaneous vaginal vs. other), arterial $\mathrm{pH}$, and smoking status [full sample is based on self-report $(n=51$ ); cotinine-validated sample is based on nonsmokers having plasma cotinine $<20 \mathrm{ng} / \mathrm{mL}(n=34)]$. 


\section{DISCUSSION}

Nonparametric analysis of epinephrine and norepinephrine concentrations showed significantly lower values for the offspring of smokers. After controlling for a number of potentially confounding variables, parametric analysis also showed that the offspring of smokers had significantly lower epinephrine concentrations at the time of delivery compared with the offspring of nonsmokers. A similar trend was seen for norepinephrine concentrations, although this finding was not statistically significant. These findings are consistent with the results of animal studies that have shown a diminished catecholaminergic response to hypoxemia as a result of prenatal tobacco exposure. One notable methodological difference is that, in our study, the catecholamine concentrations were obtained at the time of delivery, whereas, in the animal studies, the concentrations were measured during the neonatal period. Nonetheless, animal studies are useful in modeling these effects in humans, because both examine a physiologic response to hypoxic stress after prenatal nicotine exposure. In a study conducted by Slotkin et al. (7), exposure of rats to $6 \mathrm{mg} / \mathrm{kg} / \mathrm{d}$ of nicotine compared with placebo throughout gestation resulted in a deficient catecholaminergic response and greater risk of death to offspring during a postnatal hypoxic challenge. A lower catecholamine response in the nicotine-treated group would presumably impair cardiovascular responses to hypoxia, making these animals prone to cardiac arrest during a hypoxic challenge (5-7). Although other studies have also demonstrated adverse effects of prenatal nicotine exposure on autoresuscitation response (17) or arousal (18) to a hypoxic stress, catecholamine concentrations were not measured in these studies.

Of note, there may be mechanisms by which maternal smoking could affect fetal catecholamine concentrations other than what we have hypothesized. Our most robust finding was that maternal smoking was associated with reduced epinephrine concentrations in the cord blood of smokers compared with nonsmokers, regardless of whether we classified smokers by self-report or by cotinine concentration. One plausible explanation for this finding is that chronic fetal hypoxemia may differentially affect epinephrine synthesis in the offspring of smokers compared with nonsmokers. Fetuses of smokers are subjected to chronic hypoxia in utero because carbon monoxide freely crosses the placenta, binding avidly to sites that normally carry and release oxygen to the tissues (19). In sheep, chronic fetal hypoxemia is associated with a reduction of adrenal mRNA levels of the epinephrine-synthesizing enzyme phenylethanolamine $N$-methyltransferase (20). If this also occurs in humans, a reduction in phenylethanolamine $\mathrm{N}$ methyltransferase could account for our finding that maternal smoking had the greatest impact on reducing epinephrine concentrations in smokers compared with nonsmokers. Another possible explanation for a diminished catecholamine response in the offspring of smokers is that smoking chronically activates the fetal sympathetic nervous system, potentially rendering the fetus less able to respond with a catecholaminergic surge during a stressful event. In humans, it has been shown that catecholamine concentrations in the amniotic fluid of smokers are higher than in nonsmokers, which reflects chronically increased sympathetic activity (21). Fetal lambs that develop under spontaneously hypoxemic conditions also have chronically elevated catecholamine concentrations in utero; however, after birth, there is a relative blunting of adrenal medulla activation compared with fetal lambs that develop under normoxemic conditions (22). Whether the same process occurs in humans is not known.

Catecholamine concentrations observed in this study are comparable to those in previous studies of catecholamine concentrations at the time of delivery. Fetal catecholamine concentrations at birth are much higher than adult values, and the secretion of norepinephrine far exceeds that of epinephrine. Similar to a study by Lagercrantz and Bistoletti (13), we found that catecholamine release at delivery is positively correlated with gestational age but negatively correlated with arterial $\mathrm{pH}$. This correlation can be understood in terms of the fact that an infant subjected to stress (as reflected by hypoxia or acidosis) would likely release more catecholamines, but a preterm infant may be less able to respond with a catecholaminergic surge. With respect to route of delivery, our study is similar to other work that found no statistical difference in umbilical artery catecholamine concentrations between infants delivered by normal spontaneous vaginal delivery versus cesarean delivery $(13,23)$, although some studies have shown that vaginal delivery is associated with higher catecholamine concentrations (24-26).

There are some limitations to this study. First, many of our self-reported nonsmokers had cotinine concentrations that were slightly above the nonsmoking range (i.e. $30-40 \mathrm{ng} / \mathrm{mL}$ ). This discovery suggests that they may have been light smokers or that they had been exposed to environmental tobacco smoke. Because a high deception rate occurs among pregnant women who smoke, inclusion of some light smokers in the control group cannot be ruled out (27). However, the inclusion of light smokers among the nonsmoking group would mitigate against finding significant differences in catecholamine concentrations as a function of smoking condition. Future studies that identify smokers and nonsmokers at the time of delivery may benefit from the use of a spot cotinine urine dipstick, which is useful in identifying smoking status immediately. The small sample size in this study is also a limitation, resulting in differences in demographic and clinical features between groups that necessitated statistical control of potential confounding variables. Because there were few operative vaginal deliveries, this factor could not be analyzed separately; rather, a dichotomy was drawn between normal spontaneous vaginal delivery and other kinds of delivery. This dichotomy may have obscured a potential confound in the comparison of catecholamine concentrations.

The results of this study suggest that maternal smoking may affect fetal catecholamine release during a hypoxic stress. A blunted catecholamine response to hypoxemia during parturition could have an adverse effect on perinatal outcome. In the context of a prospective study, our results could provide a mechanistic underpinning for the increased perinatal morbidity and mortality associated with maternal smoking during pregnancy. 
Future research should determine the reliability of this association by studying these variables in larger groups of women of similar gestational age, without concurrent medical problems, and whose offspring were born via a similar route of delivery. Finally, longitudinal research designs are needed to evaluate the predictive validity of catecholamine concentrations at birth in relation to neonatal outcomes, including SIDS.

Acknowledgments. The authors thank Kristen Sandstrom for coordinating this study, Jeffrey Van Kirk for statistical analyses, and Donna Rapheal for cotinine analyses.

\section{REFERENCES}

1. DiFranza JR, Lew RA 1995 Effect of maternal cigarette smoking on pregnancy complications and sudden infant death syndrome. J Fam Pract 40:385-394

2. Haglund B, Cnattingius S 1990 Cigarette smoking as a risk factor for sudden infant death syndrome: a population-based study. Am J Public Health 80:29-32

3. Anderson HR, Cook DG 1997 Passive smoking and sudden infant death syndrome: review of the epidemiological evidence. Thorax 52:1003-1009

4. Sullivan FM, Barlow SM 2001 Review of risk factors for sudden infant death syndrome. Paediatr Perinatal Epidemiol 15:144-200

5. Lagercrantz H, Slotkin TA 1986 The stress of being born. Sci Am 254:100-107

6. Slotkin TA 1998 Fetal nicotine or cocaine exposure: which one is worse? J Pharmacol Exp Ther 285:931-945

7. Slotkin TA, Lappi SE, McCook EC, Lorber BA, Seidler FJ 1995 Loss of neonatal hypoxia tolerance after prenatal nicotine exposure: implications for sudden infant death syndrome. Brain Res Bull 38:69-75

8. Gregg AR, Weiner CP 1993 "Normal" umbilical arterial and venous acid-base and blood gas values. In: Pitkin RM, Scott JR (eds) Clinical Obstetrics and Gynecology. JB Lippincott, Hagerstown, MD, pp 24-32

9. Benowitz NL, Kuyt F, Jacob III P, Jones RT, Osman AL 1983 Cotinine disposition and effects. Clin Pharmacol Ther 34:604-611

10. Riley RJ, Johnson JWC 1993 Collecting and analyzing cord blood gases. In: Pitkin RM, Scott JR (eds) Clinical Obstetrics and Gynecology. JB Lippincott, Hagerstown, MD, pp 13-23
11. Kilts CD, Gooch MD, Knopes KD 1984 Quantitation of plasma catecholamines by on-line trace enrichment high performance liquid chromatography with electrochemical detection. J Neurosci Methods 11:257-273

12. Jacob III P, Wilson M, Benowitz NL 1981 Improved gas chromatographic method for the determination of nicotine and cotinine in biologic fluids. J Chromatogr 222:61-70

13. Lagercrantz H, Bistoletti P 1973 Catecholamine release in the newborn infant at birth Pediatr Res 11:889-893

14. Bigger HR, Silvestri JM, Shott S, Weese-Mayer DE 1998 Influence of increased survival in very low birth weight, low birth weight, and normal birth weight infants on the incidence of sudden infant death syndrome in the United States: 1985-1991. J Pediatr 133:73-78

15. Cohen J 1988 Statistical Power for the Behavioral Sciences, 2nd ed. Lawrence Erlbaum Associates, Hillsdale, NJ, pp 24-27

16. Jarvis M, Tunstall-Pedoe H, Feyerabend C, Vesey C, Salloojee Y 1984 Biochemical markers of smoke absorption and self-reported exposure to passive smoking. J Epidemiol Community Health 38:335-339

17. Fewell JE, Smith FG 1998 Perinatal nicotine exposure impairs ability of newborn rats to autoresuscitate from apnea during hypoxia. J Appl Physiol 85:2066-2074

18. Lewis KW, Bosque EM 1995 Deficient hypoxia awakening response in infants of smoking mothers: possible relationship to sudden infant death syndrome J Pediatr 127:691-699

19. Longo LD 1977 The biological effects of carbon monoxide on the pregnant woman, fetus, and newborn infant. Am J Obstet Gynecol 129:69-103

20. Adams MB, Phillips ID, Simonetta G, McMillen IC 1998 Differential effects of increasing gestational age and placental restriction on tyrosine hydroxylase, phenylethanolamine $\mathrm{N}$-methyltransferase, and proenkephalin A mRNA levels in the fetal sheep adrenal. J Neurochem 71:394-401

21. Divers WA, Wilkes MM, Babaknia A, Yen SSC 1981 Maternal smoking an elevation of catecholamines and metabolites in the amniotic fluid. Am J Obstet Gynecol 141:625-628

22. Smolich JJ, Esler MD 1999 Total body catecholamine kinetics before and after birth in spontaneously hypoxemic fetal lambs. Am J Physiol 277:R1313-R1320

23. Eliot RJ, Lam BS, Leake RD, Hobel CJ, Fisher DA 1980 Plasma catecholamine concentrations in infants at birth and during the first 48 hours of life. J Pediatr 96:311-315

24. Puolakka J, Kauppila A, Tuimala R, Jouppila R, Vuori J 1983 The effect of parturition on umbilical blood plasma levels of norepinephrine. Obstet Gynecol 61:19-21

25. Jones CM, Greiss FC 1982 The effect of labor on maternal and fetal circulating catecholamines. Am J Obstet Gynecol 144:149-153

26. Irestedt L, Lagercrantz H, Hjemdahl P, Hagnevik K, Belfrage P 1982 Fetal and maternal plasma catecholamine levels at elective cesarean section under general or epidural anesthesia versus vaginal delivery. Am J Obstet Gynecol 142:1004-1010

27. Boyd NR, Windsor RA, Perkins LL, Lowe JB 1998 Quality of measurement of smoking status by self-report and salivary cotinine among pregnant women. Matern Child Health J 2:77-83 